\title{
¿Trabajo decente o trabajo indecente?
}

\author{
Julio Bordas Martínez \\ Universidad Nacional de Educación a Distancia, Madrid, España \\ jbordas@poli.uned.es \\ Francisco Javier Pinilla García \\ Universidad Nacional de Educación a Distancia, Madrid, España \\ jpinilla@poli.uned.es
}

\begin{abstract}
Resumen: El trabajo decente, tal como lo define la OIT, es una forma digna de trabajar en un contexto de políticas de generación de empleo, con la capacidad de exigir judicialmente los derechos de los trabajadores, en el marco de una política institucional de protección social y con el reconocimiento de una negociación colectiva autonormativa consolidada. A estos cuatro objetivos tradicionales debemos sumar el objetivo transversal de la igualdad de género. El desempleo, el subempleo y el empleo precario, en el marco de la ideología de la flexiseguridad, configuran el caldo de cultivo del empleo indecente o empleo precario.
\end{abstract}

Palabras clave. Trabajo decente, derechos de los trabajadores, desempleo, informalidad.

\begin{abstract}
Decent work, as defined by the ILO, is a worthy way of working, in a context of employment generation policies, with the capacity to legally demand the rights of workers, within the framework of an institutional policy of social protection and with the recognition of a consolidated autonomous collective bargaining. To these four traditional objectives we must add the transversal objective of gender equality. Unemployment, underemployment and precarious employment, within the framework of the ideology of flexicurity, are the breeding grounds of indecent employment or precarious employment.
\end{abstract}

Keywords. Decent work, workers rights, unemployment, informality. 


\section{INTRODUCCIÓN}

El concepto de trabajo decente es impreciso, utópico si se quiere, pero muy importante, y debemos su enunciado a Juan Somavia, presidente de la Organización Internacional del Trabajo, quien lo definió durante su 87. ${ }^{a}$ Conferencia, en junio de 1999, y que desde entonces se viene celebrando el 7 de octubre de cada año (OIT, 1999).

El trabajo decente es una traducción literal de decent work, que al pasar al español se le presume una carga moral extraordinaria, ya que en inglés no es de semejante categoría; decent work vendría a significar en español trabajo adecuado o, mejor aún, trabajo digno; de tal manera que lo indecente no es el trabajo realizado, sino la forma de ejecutarlo.

En coherencia con lo anterior, trabajo indecente no sería trabajo inmoral, sino trabajo inadecuado o, más concretamente, trabajo precario, caracterizado por falta de seguridad en el empleo, falta de seguridad en el salario, falta de seguridad en las prestaciones sociales, falta de seguridad en la sindicación, el diálogo social y la negociación colectiva.

Además de impreciso, el concepto de trabajo decente es un enunciado utópico en el sentido de que es muy positivo idealmente, pero resulta materialmente inalcanzable en todo tiempo, lugar y sector.

El carácter idealmente positivo de la reivindicación universal del trabajo decente es nueva en la forma de enunciarlo y en su denominación tan llamativa, pero no es nueva como reivindicación universal en el sentido de considerar que un trabajo decente es, en primer lugar, un trabajo libre, remunerado, dependiente y ajeno y, en segundo lugar, en tanto que decente o digno, es una forma de trabajar dentro de un marco caracterizado por cuatro aspectos: una política de generación de empleo, la garantía judicial de los derechos de los trabajadores, un sistema público de protección social y un sistema institucionalizado de diálogo social entre empresarios y trabajadores, con capacidad autonormativa, y respaldo de las instituciones públicas (tripartismo); y a los que debemos añadir un eje trasversal que estaría relacionado con una política de igualdad de género.

El carácter utópico se revela evidente cuando vemos, aún hoy día, la prevalencia del trabajo esclavo (Figura 1), del trabajo infantil (Figura 2), del reclutamiento militar de niños, de la explotación sexual de las mujeres, de la explotación laboral de los inmigrantes (244 millones en 2015), de la falta de empleo estable con un salario suficiente, de las condiciones de trabajo insalubres y peligrosas, de la falta de libertad sindical, de la imposibilidad de conciliar la vida laboral con la familiar, etc. 


\section{FIGURA 1}

El trabajo esclavo en el mundo

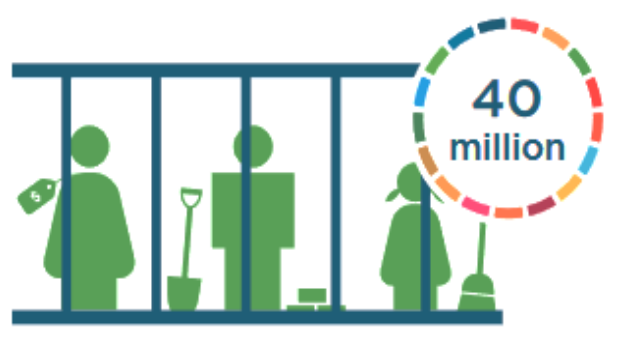

\section{METRICS}

40 million people were victims of modern slavery. This includes:

- 25 million people in forced labour

- 15 million people in forced marriage

\section{PREVALENCE}

There were 5.4 victims of modern slavery for every thousand people in the world in 2016.

There were 5.9 adult victims of modern slavery for every 1,000 adults in the world and 4.4 child victims for every 1,000 children in the world.
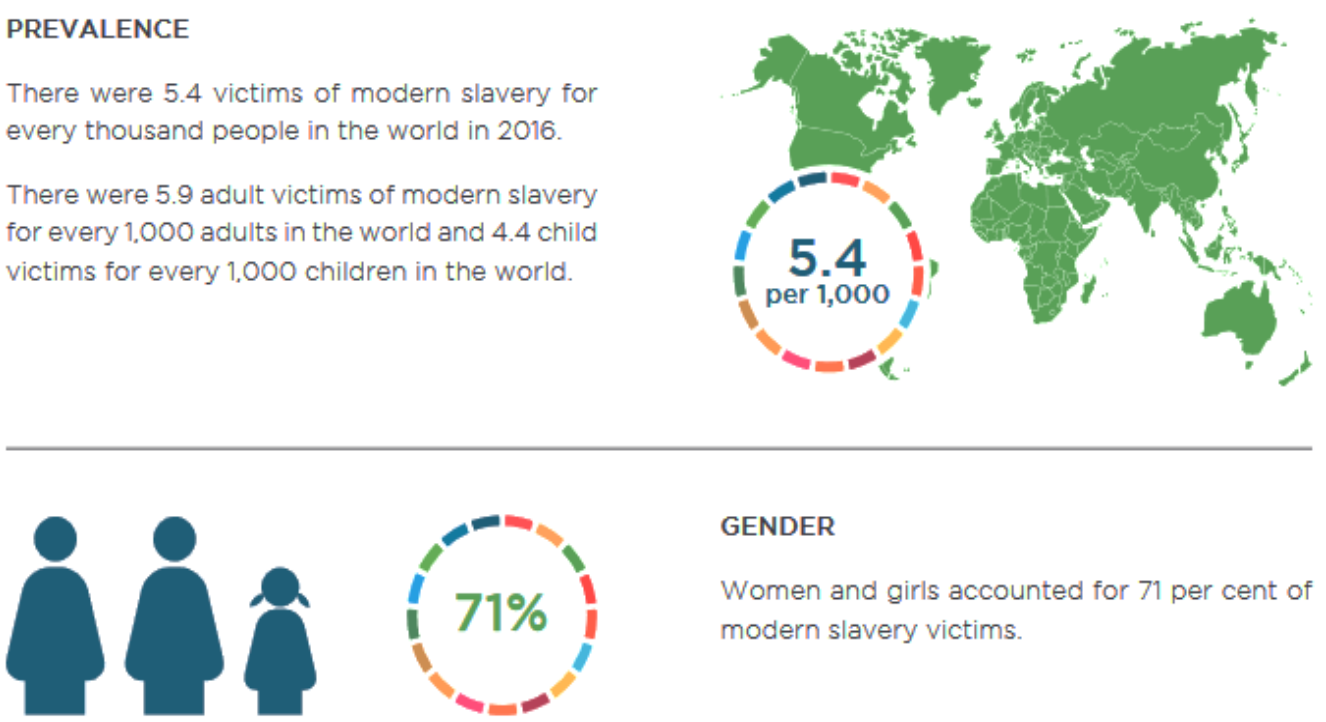

\section{GENDER}

Women and girls accounted for 71 per cent of modern slavery victims.

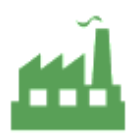

\section{DEBT BONDAGE}

Debt bondage affected half of all victims of forced labour imposed by private actors.

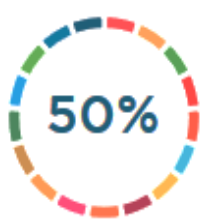

Fuente: OIT (2017: 5).



CHILDREN



One in four victims of modern slavery were children. 
En cualquier caso, el trabajo decente, como aspiración a un trabajo digno universal, no es una reivindicación novedosa, es una nueva forma de enunciarlo.

FIGURA: 2

Trabajo infantil en el mundo

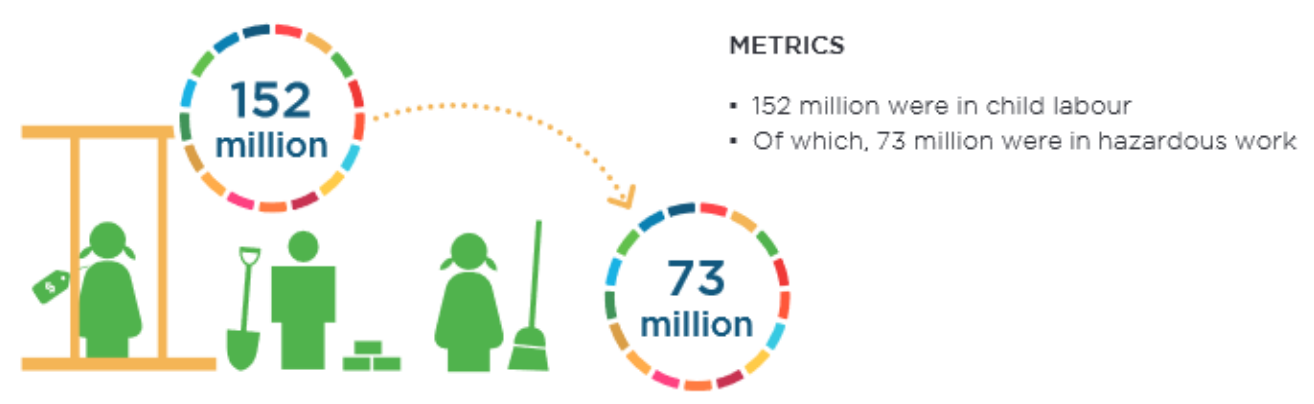

\section{REGIONAL PREVALENCE OF CHILD LABOUR}

$\begin{array}{ll}\text { - Africa } & 19.6 \% \\ \text { - Americas } & 5.3 \% \\ \text { - Arab States } & 2.9 \% \\ \text { - Asia and the Pacific } & 7.4 \% \\ \text { - Europe and Central Asia } & 4.1 \%\end{array}$

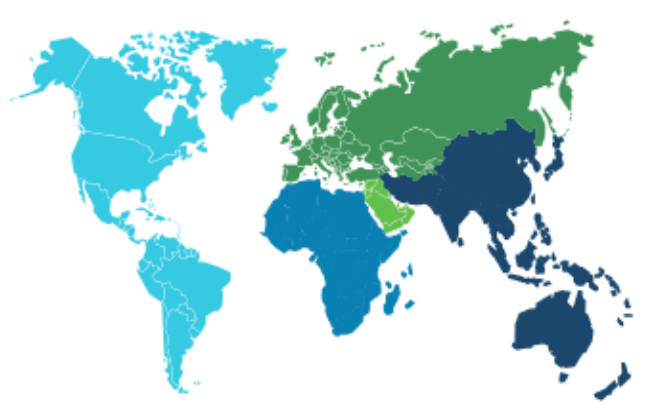

\section{OF THE 152 MILLION CHILDREN IN CHILD LABOUR}

\section{AGE PROFILE}

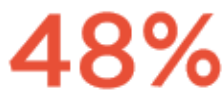

5-11 years-olds

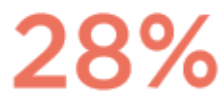

12-14 years-olds



GENDER

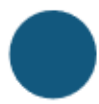

$58 \%$

88 million

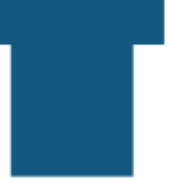



ECONOMIC ACTIVITY
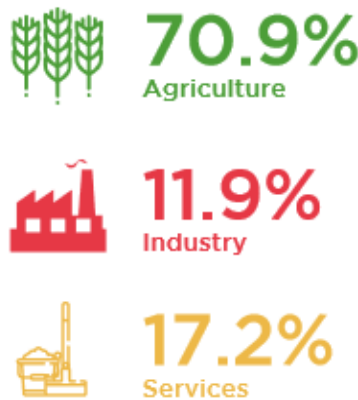

Fuente: OIT (2017: 5). 
La reivindicación del trabajo decente ya figuraba sustancialmente en el Tratado de Paz de Versalles, de junio de 1919, hace un siglo exactamente, en el que además de poner fin a la I Guerra Mundial, haciéndose responsable de la misma al Imperio alemán, se creaba la Sociedad de Naciones y la Organización Internacional de Trabajo.

En su art. 427, el Tratado de Versalles ya exigía que el trabajo dejara de considerarse una mercancía, que se prohibiera el trabajo infantil, se garantizara un salario justo para la manutención de la familia, se garantizara el mismo salario por el mismo trabajo sin discriminación por sexo, se estableciera una jornada de ocho horas diarias y 48 semanales, con un día completo de descanso a la semana, se garantizara la libertad sindical, etc., etc.

En la Declaración de Filadelfia de mayo de 1944 la OIT ya declaraba que el trabajo no era una mercancía, que todos los trabajadores tenían derecho a la igualdad de oportunidades sin discriminación por razón de religión, raza o sexo, se exigía la libertad de expresión y asociación para la defensa de sus intereses y el reconocimiento normativo de la negociación colectiva, la búsqueda del pleno empleo para la erradicación de la pobreza y la defensa de unas condiciones de trabajo saludables con sueldos y jornadas justas.

La Declaración Universal de los Derechos Humanos de diciembre de 1948, en sus arts. 23 y ss., establecía el derecho a la libertad de trabajo sin discriminación, al salario justo y suficiente, al descanso y a las vacaciones pagadas, a la protección social y a la libertad sindical.

En consecuencia, sin que sea necesario llegar a la Constitución Española de diciembre de 1978, ni al Estatuto de los Trabajadores de marzo de 1980, ni a la Carta Comunitaria de los Derechos Sociales de diciembre de 1989, ni al tratado de Maastricht de febrero de 1992, queda claro que las reivindicaciones actuales que configuran el trabajo decente: política de empleo, derechos laborales, protección social y diálogo social, más el eje transversal de la igualdad de género, ya existían hace tiempo y ahora se subrayan y se potencian en el marco de la Agenda 2030 de Naciones Unidas, aprobada en septiembre de 2015, cuyo Plan de Implementación aprobó la OIT en agosto de 2016, concretándose en España en el segundo informe sobre el Plan Director por un Trabajo Digno, aprobado por el Gobierno en julio de 2018 (Gobierno de España, 2019).

\section{EL TRABAJO INDECENTE O PRECARIO}

La existencia generalizada y mayoritaria de trabajo indecente o precario en la mayoría de los países y en gran número de sectores es lo que justifica que cien años después del Tratado de Versalles se siga reivindicando un trabajo decente, y esto se debe a que la 
fuente misma del trabajo está contaminada, y solo controlando y depurando el trabajo indecente se obtendrá trabajo decente.

Hay tres circunstancias relacionadas con el empleo asalariado que, más allá de la alienación o la sustracción de plusvalía, que no estamos analizando en este momento, lo enturbian, generando trabajo asalariado de mala calidad, trabajo precario, y estas son: el desempleo, el empleo informal y el subempleo.

La primera circunstancia propiciadora del trabajo precario es el desempleo masivo, que afecta a los países subdesarrollados, y el desempleo estructural de los países desarrollados, que afecta especialmente a jóvenes en busca de su primer empleo y a parados mayores de cuarenta y cinco, y que no solo no se va resolviendo, sino que se va acrecentado por la deslocalización de determinadas cadenas de producción, por las reconversiones industriales que no condujeron a la recolocación y por la innovación tecnológica, telecomunicaciones y robótica incluidas, cuya extensión es desigual pero su crecimiento universal (Figura 3), que hacen prescindibles a millones de trabajadores y oficios enteros, provocando movimientos de población (Figura 4) que terminan componiendo bolsas de pobreza que se establecen muchas veces en barrios concretos de algunas ciudades, hacinados, donde componen guetos cerrados, muy homogéneos internamente pero muy heterogéneos respecto de los demás, o slams, multiculturales y conflictivos, a los que han llegado por decantación de un proceso de vulnerabilidad social, que derivó en exclusión social, para terminar consolidándose como marginación social, y sobrevivir de la delincuencia y/o de la asistencia social, sin muchas expectativas de futuro y próximos a la anomia.

En el caso español y por lo que al paro juvenil se refiere:

Los años de la burbuja inmobiliaria [como nos recuerda Monereo] invitaron a numerosos jóvenes a abandonar el sistema formativo para adentrarse en un mercado de trabajo descualificado, pero que en su momento era altamente dinámico. De este modo, no solo nos enfrentamos a altísimas tasas de desempleo juvenil, sino además a importantes carencias formativas de muchos de los trabajadores desempleados [Monereo, 2018: 15].

La existencia de este desempleo masivo, que supone el $5 \%$ de los activos de todo el planeta, según la OIT (2019), no solo compone un ejército de reserva del capital que permite a los empresarios ofrecer empleos en malas condiciones, que siempre hay quien las acepta por pura necesidad, sino que también produce un efecto de estigmatización, no solo para los parados sin futuro que se saben prescindibles, en los términos de Ulrich Beck (2005), sino que produce una especie de prevención general negativa que amedrenta al resto de los trabajadores para que acepten empleos informales o subempleos para no verse como ellos. 


\section{FIGURA 3}

Evolución de la robotización en el mundo. Densidad de los robots en la manufactura.

Cantidad de robots industriales multipropósito por cada 1.000 personas empleadas en el sector de la manufactura

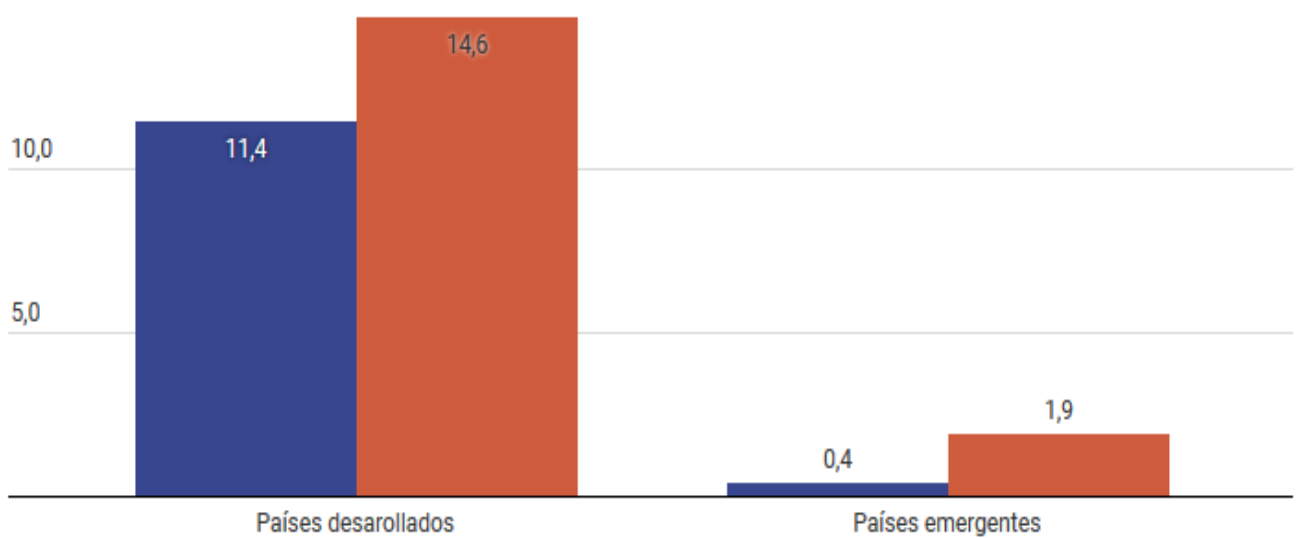

Fuente: OIT (2019).

\section{FIGURA 4}

Potencial del flujo migratorio mundial. Proporción de participantes que se mudarían de manera permanente a otro país por grupos de edad, 2016

Jóvenes (15-24) $\quad$ Adultos (25-64)

40

30

20

10



Fuente: OIT (2015).
43

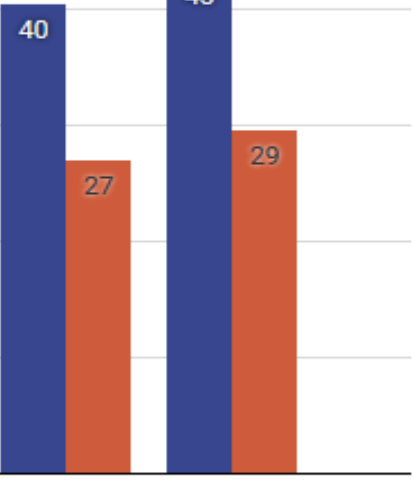

América

Latina y el 
La escasez de empleos crea ese "ejército de reserva" que posibilita ofertar empleos cada vez menos retribuidos y más exigentes en el esfuerzo y compromiso demandado por la empresa, denominados precarios. La precariedad laboral y el empleo informal han ido extendiéndose en España a partir de la primera reforma laboral del año 1984 por la que se instaura la figura del trabajador temporal, rompiendo así la "norma social de empleo" anterior (Prieto, 2002).

Después del desempleo, el segundo acicate del empleo precario es el empleo informal, que es la remercantilización del trabajo; se trata de un trabajo mercancía, persona del trabajador incluida, que desempeña una tarea en el tiempo y lugar demandados por el empleador, y que deja de prestarlo cuando no lo necesitan. No se trata de que no se le pague, es más, puede que paguen más de lo que suelen pagar a empleados de igual categoría y especialización; de lo que se trata es de que les pagan cuando los usan, sin que disfruten de derechos laborales, sin estabilidad en el empleo, sin jornadas justas, sin sus correspondientes vacaciones pagadas, ni seguridad social, ni asistencia sanitaria, ni seguros de desempleo y de jubilación; y a veces sin contrato o con contratos mercantiles como si de una compraventa se tratara y como si empleado y empleador estuvieran en igualdad de condiciones, que, naturalmente, no es el caso. El empleo informal se da en un amplio campo de la estructura económica, la economía informal, que tiene su origen conceptual en la economía de los países subdesarrollados sobre los que hablaba Hart, antropólogo que acuñó el término, pero que está presente en las economías desarrolladas, como nos explica Alejandro Portes (2013: 177):

Encontramos la economía informal en actividades habituales, como cuando compramos un reloj barato [...] Cuando llamamos a alguien para que nos haga algún arreglo en casa y le pagamos sin factura o cuando contratamos a una mujer inmigrante para que limpie la casa [...] La industria de la confección que produce la ropa que compramos y usamos tiene frecuentemente al otro extremo de la cadena de producción talleres clandestinos [...] El personal de trastienda que realiza buena parte de la limpieza y preparación de la comida en los restaurantes está formado por inmigrantes, a menudo recién llegados y sin documentación en regla... La industria informática que produce los portátiles que utilizamos es conocida por subcontratar el montaje [...] Incluso a personas que trabajan desde casa [... y esto es así porque...] Las actividades informales, definidas como aquellas que se producen fuera del ámbito de la regulación del Estado, pueden estar encaminadas, en primer lugar, a la supervivencia del individuo o su familia mediante la producción directa o la simple venta de bienes y servicios para su subsistencia. En segundo lugar, pueden ir destinadas a aumentar la flexibilidad en la gestión y a reducir los costes laborales de las empresas del sector formal mediante la contratación y subcontratación irregular de empresarios informales. En tercer lugar, pueden haber sido organizadas por pequeñas empresas para acumular capital mediante la movilización de sus lazos de solidaridad, el aumento de la flexibilidad y la disminución de costes. 
El empleo informal, aunque ciertamente cumple algunas funciones económicas, además de no generar derechos para el trabajador, tampoco contribuye a la comunidad, dificultando la financiación de servicios públicos como la educación, el transporte, la sanidad o la seguridad públicas, que utiliza todo el mundo, incluidos los que no contribuyen a su mantenimiento.

El empleo informal se sostiene por la pericia y actualización de los trabajadores, por sus relaciones entre ellos, que muchas veces se conocen y se "sindican", por la innovación tecnológica que permite deslocalizar y destemporalizar el trabajo realizado y por la necesidad de los empleadores en determinados momentos, lugares o sectores; muchas veces puntuales y perentorios, para atender demandas masivas estacionales que si no se satisfacen, favorecen a la competencia y amenazan con sacarlos del mercado.

Por último, el subempleo es el otro gran caldo de cultivo del empleo indecente y, por resumir, tiene cuatro manifestaciones: contrato temporal, jornada reducida, salario escaso y empleo inadecuado para las competencias del trabajador.

La temporalidad en el empleo respecto del empleo fijo no hace referencia a que el salario sea escaso o la jornada extenuante; hace referencia a que la duración del contrato de trabajo es temporal, lo que impide al trabajador planificar su futuro y, además, aumenta los riesgos laborales por falta de formación y de experiencia en el desempeño de sus tareas. El trabajo temporal en España supone casi el 27\% de los contratos, lo que significa que, junto con Polonia, ocupamos la tasa de temporalidad laboral más alta de la Unión (INE, 2019) (Figura 5).

\section{FIGURA 5}

Temporalidad y parcialidad en España.

Evolución de las tasas de temporalidad y parcialidad

40

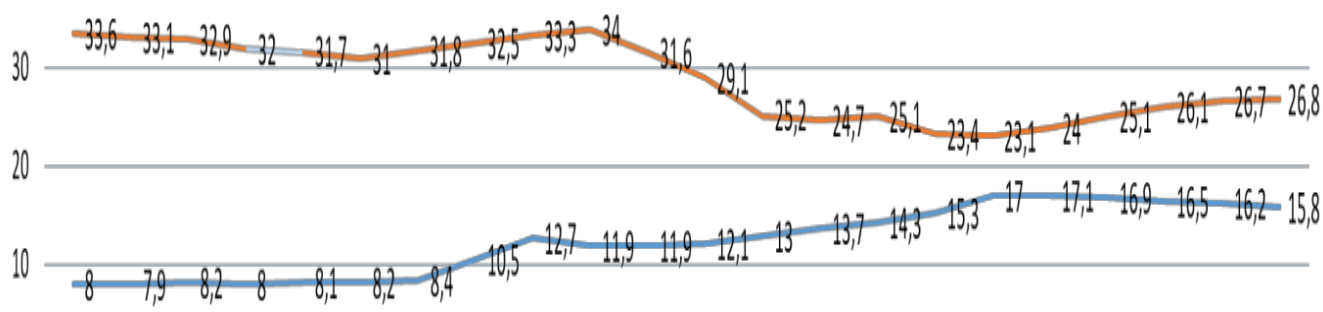

0

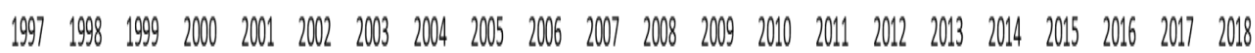

$$
\text { - tasa parcialidad - tasa temporalidad }
$$

Fuente: Ministerio de Trabajo, Migraciones y Seguridad Social (2019). 
Otro tipo de subempleo es el que proporciona una jornada reducida, de pocas horas al día, muchas veces móviles en el tiempo y en el espacio, de forma que el empleador solo remunera la escasa jornada trabajada y utiliza al trabajador cuando estrictamente lo necesita, ya sea en una instalación u otra, ya sea en turnos de mañana, tarde o noche (Figura 6).

\section{FIGURA 6}

Evolución del tiempo parcial por género en España.

Porcentaje de trabajadores a tiempo parcial sobre el total de asalariados

\begin{tabular}{lcrrrr}
\hline & $\mathbf{1 9 9 6}$ & $\mathbf{2 0 1 3}$ & $\mathbf{2 0 1 6}$ & $\mathbf{2 0 1 7}$ & $\mathbf{2 0 1 8}$ \\
\hline Hombres & \multicolumn{1}{c}{3} & 6,6 & 8,3 & 7,8 & 7,2 \\
Mujeres & 16,6 & 24,8 & 25,4 & 25,4 & 25,1 \\
Total & 7,7 & 15,3 & 16,5 & 16,2 & 15,8 \\
\hline
\end{tabular}

Fuente: Ministerio de Trabajo, Migraciones y Seguridad Social (2019).

Que el trabajador no gane lo suficiente para mantener a su familia y llegar a fin de mes a pesar de estar ocupado toda la jornada es lo que define al trabajador pobre, que no puede mantener a su familia y tampoco tiene tiempo para formarse o para buscar otro trabajo: está atado por un salario insuficiente.

Que la jornada sea escasa y variable dificulta la conciliación de la vida familiar y laboral e impide tanto prosperar en el trabajo como buscarse otro empleo con mejor jornada y vacaciones incluidas.

Un último tipo de subempleo es aquel que se caracteriza por la utilización de trabajadores sobrecualificados para desempeñar tareas menores, lo que, sin duda, conduce a la insatisfacción en el trabajo y a tomarse la tarea como una especie de impuesto temporal que permite, a cambio de hacer lo que no gusta, poder ir sobreviviendo junto a la familia.

El caso del empleo inadecuado a las competencias del trabajador es mucho más frecuente de lo que se piensa, y podemos encontrarlo preferentemente entre los trabajadores inmigrantes y entre los funcionarios.

Entre los trabajadores inmigrantes es fácil encontrar a médicas haciendo de enfermeras, enfermeras trabajando de limpiadoras, ingenieros trabajando de cocineros y oficiales del ejército de su país cuidando ancianos en residencias o montando calderas de calefacción; pianistas y cantantes trabajando de taxistas y profesores universitarios en países del África subsahariana trabajando de barrenderos, por ejemplo. 
Entre los funcionarios es bastante frecuente encontrar licenciados ocupando puestos de grupo $\mathrm{C} 1$ con nivel 15, y eso se debe a que en las oposiciones hay requisitos mínimos (bachiller para el grupo C) pero no un tope máximo por arriba, por lo que licenciados, ingenieros y arquitectos pueden conseguir plaza en ayuntamientos, hospitales o la Policía, aprovechando su formación y hábitos de estudio, pero permaneciendo durante años en puestos insatisfactorios en los que no pueden aplicar los conocimientos que obtuvieron en la universidad.

En definitiva, el desempleo, el empleo informal y el subempleo componen un caldo de cultivo que contamina, por mezcla, el trabajo decente, y, además, tiene defensores que lo justifican por las necesidades del mercado y por las ventajas que estos empleos proporcionan a las empresas y a los trabajadores. Se trata de la coartada de la "flexiseguridad" (Falguera, 2007), que defiende el trabajo flexible en el tiempo, el espacio y los distintos sectores de forma que los trabajadores de determinado nivel y especialización estén disponibles, como las mercancías en una organización del trabajo just in time, esperando que la parte de seguridad en el empleo la aporte el mercado mismo de forma que cuando un trabajador cese en una actividad, en cualquier empresa o sector, se vaya a realizarla a otra que circunstancialmente lo necesite. El trabajador pierde sus derechos laborales y pone su esperanza en la oferta y la demanda.

El teletrabajo completo, permanente y obligatorio, que la OIT (2002) denomina "periférico", es el que Carnoy (2001) denomina trabajo flexible, entendiendo por tal la subordinación del trabajo al mercado, de forma que los cometidos laborales y la jornada se adapten constantemente a los productos, procesos y mercados. Por ello, las empresas exigen a sus empleados una cualificación superior, mayor capacidad de autoprogramarse, responsabilidad individual y disposición a seguir planes flexibles y jornadas prolongadas. No obstante, y a pesar de estas exigencias, las empresas aflojan los lazos que las ligan a los trabajadores porque su objetivo es lograr mano de obra just in time que les permita aumentar el número de horas cuando aumente la demanda y reducirlas cuando caiga. En la práctica, esto quiere decir más trabajo eventual y menos derechos para los trabajadores.

Por el contrario, el teletrabajo parcial y voluntario, que la OCDE denomina "flexibilidad funcional" (OCDE, 1999: 420) es propio del personal "esencial”, que, como explica Albizu (1997), es el necesario para el cumplimiento del objeto social de la compañía. Este personal suele dedicarse a la empresa a tiempo completo y con un grado de libertad proporcional a su responsabilidad.

El teletrabajo funcional permite a los trabajadores disponer de una jornada flexible en lugar de ser su trabajo una mercancía flexible. 
Disponer de una jornada realmente flexible supone para muchos de ellos una enorme ventaja funcional, por cuanto que pueden desempeñar en el hogar algunas tareas productivas sin tener que desplazarse o desatender a sus familias.

Cuando esta flexibilidad funcional se combina con la innovación tecnológica se produce no solo un incremento considerable y sostenido del empleo, sino también una mejora en la calidad de vida en el trabajo.

Un ejemplo de aplicación de este tipo de teletrabajo lo podemos encontrar en la AT\&T (Apgar, 2002), que ha demostrado un ahorro de 460.000 dólares al año gracias a proporcionar a parte de su personal "esencial" ordenadores portátiles y teléfonos móviles. De esta manera, una parte de sus tareas las desempeñan en su hogar o en la oficina del cliente y la otra parte, en la empresa y en espacios comunes polivalentes, organizados como un "trabajo hotel" con el que el trabajador debe ponerse en contacto a través de un "recepcionista" para reservar hora, día o semana y advertir de los equipos que vaya a utilizar durante su estancia en la oficina.

Esta flexiseguridad tiene defensores importantes, que son los que redactaron y publicaron el Libro Verde en noviembre de $2006^{1}$ (Comisión de la Comunidades Europeas, 2006), por encargo de la Unión Europea, al considerar que la innovación tecnológica, la globalización, la externalización y la sostenibilidad medioambiental exigían un trabajo flexible de forma que el incremento de inseguridad de los trabajadores redundara en beneficio de la competitividad de las empresas, con el pretexto de que así generarían más empleo y los trabajadores competentes y productivos siempre tendrían quien los contratase en algún tiempo, lugar y sector.

Los trabajadores se convertirían así en proveedores de sus propios servicios laborales, una especie de ETT unipersonal.

Esta flexiseguridad (Gil y Gil, 2008) supone una remercantilización del trabajo, una desindicalización del trabajo, con un generalizado desprestigio y desafección de los sindicatos, una postergación de la negociación colectiva autonormativa y tripartita, y anula la requerida flexibilidad en la jornada laboral para conciliar con la vida familiar, que se sustituye por la flexibilidad en el empleo, que convierte a todos los trabajadores en unos autónomos subcontratistas a destajo.

Como señala Miguélez (2002: 44), la persecución incansable de la competitividad a través de la flexibilidad de la mano de obra requiere, en primer lugar,

${ }^{1}$ Libro Verde para la modernización del derecho laboral para afrontar los retos del siglo XXI, 2006". https://eur-lex.europa.eu/legal-content/ES/TXT/?uri=celex:52006DC0708 
... debilitar al trabajador individualmente por cuanto desde el momento en que se sienta inseguro sus demandas serán menores, más tímidas y cautelosas; siendo así, la estrategia del trabajador individual para permanecer en el empleo tendrá en cuenta los riesgos que implica plantear exigencias que supuestamente la empresa no quiera o no pueda aceptar. Pero también significa debilitar al trabajador como sujeto colectivo y a su expresión- el sindicato o el comité o la representación social en general- porque supone debilitar su base.

Hay algunos datos que nos indican el grado de trabajo indecente o precario que se da en el mundo, siendo conscientes de que las cifras de precariedad de las que disponemos probablemente sean más bajas que las reales, porque en muchos países no hay datos disponibles, en unos porque no existen, y, en otros, en los que sí existen, porque están tan maquillados que terminan alterando el cómputo promedio; además, hay fuentes que con todo el rigor metodológico del que son capaces ofrecen datos diferentes sobre el mismo indicador. Piénsese, por ejemplo, en la diferencia que hay entre los datos que sobre la brecha salarial entre hombres y mujeres proporciona la CEOE, CC. OO., Cáritas, Eurostat o la OIT.

Con estas salvedades, podemos decir que hay unos 3.500 millones de población activa mundial (Banco Mundial, 2019), cuya mayoría trabaja a tiempo parcial (60\%), de los que 200 millones están parados (algo así como la población de Pakistán), de los cuales el $37 \%$ son jóvenes en busca de su primer empleo.

Dos mil millones de trabajadores están ocupados informalmente, algunos de ellos solapándose con los parados, y 1.300 millones están ocupados formalmente, aunque el $50 \%$ de ellos no dispongan de seguridad social, a pesar de haber unos dos millones y medio de fallecidos por accidentes y enfermedades laborales al año, y la brecha salarial entre hombres y mujeres sea del $20 \%$, tanto a escala planetaria como en los países más desarrollados con empleos formales y estadísticas más rigurosas, en los que las mujeres también suelen ganar un 20\% menos que sus compañeros varones por realizar un trabajo similar. Suecia es el país con una menor brecha salarial después de Filipinas, donde, anecdóticamente, la brecha salarial es de un 10\% a favor de la mujer (OIT, 2019). Como subraya M. Teresa Igartúa, "para lograr la plena igualdad entre hombres y mujeres es necesario modificar el papel tradicional, tanto del hombre como de la mujer en la salud y en la familia" (Igartúa, 2018: 393).

Otra brecha es la digital, que puede llegar a escala planetaria a un 50\% entre unos hogares y otros, teniendo en cuenta que se trata de un porcentaje promedio cuya desviación típica debe de ser amplia, es decir, que en determinados países, más atrasados que 
pobres, puede disponerse de un equipamiento informático muy inferior al 50\% y en otros países más avanzados se sobrepase con creces.

A este marco general podemos añadir algunas pinceladas dramáticas, como que haya mil millones de pobres de solemnidad que subsisten con menos de dos dólares al día (Banco Mundial, 2019), la inmensa mayoría residentes en África y Asia; que contemos con 250 millones de migrantes desarraigados, 170 millones de niños trabajando y 25 millones de esclavos, casi la mitad de ellos migrantes y la cuarta parte mujeres explotadas sexualmente.

En el caso concreto de España (Fundación $1^{\circ}$ de Mayo, 2019a), país desarrollado de la Unión Europea, el trabajo indecente o precario tampoco escasea.

La población activa alcanza los 23 millones de personas, de las cuales 19,7 millones están ocupados, aunque 13 millones de los trabajadores ocupados sean pobres, es decir, se encuentren por debajo del umbral de la pobreza, establecido para España en 8.000 euros al año por persona, en 12.800 para dos personas y en 18.000 para una familia de cuatro personas.

El 50\% del conjunto de los trabajadores ocupados no tiene representación sindical, el $30 \%$ dispone de contratos de trabajo temporal y el 33\% trabaja en fines de semana y festivos.

De la población activa señalada, 3,3 millones están desempleados, lo que supone una tasa de paro del 15\% (Fundación $1^{\circ}$ de Mayo, 2019), que viene a doblar la tasa de paro media de la Unión Europea, solo superada por Grecia y seguida por Italia. Estos parados disponen de cobertura por desempleo solo en el 50\% de los casos.

La brecha salarial entre hombres y mujeres en España es del 20\%, aproximadamente, según cómo se haga el cómputo, y a esta minusvaloración del mismo tipo y cantidad de trabajo realizado en comparación con un varón hay que añadir que el $80 \%$ de todas las tareas domésticas y asistenciales realizadas en el hogar y no presupuestadas las realizan las mujeres. Ello hace que las mujeres trabajadoras dediquen casi el doble de horas semanales que los varones a tareas no remuneradas (Figura 7).

Teniendo en cuenta el precio medio de la hora de trabajo realizado por empleadas de hogar, se estima que el trabajo no remunerado realizado por las mujeres en el hogar y en labores asistenciales relacionadas con la dependencia supondría cerca del $35 \%$ de PIB de España, situado en 1,3 billones aproximadamente, poco más que el de México y poco menos que el de Italia, y parecido al de Canadá y Australia.

A todas estas características relacionadas con el trabajo precario en España habría que añadir que el 10\%, aproximadamente, de la actividad laboral se desarrolla dentro de la esfera del empleo informal, sin contrato, sin seguros, sin estabilidad, sin derecho, sin sindicatos y sin impuestos. 


\section{FIGURA 7}

Duración en horas de la jornada semanal de trabajo según género, 2015



Empleo principal $\square$ Otro empleo $\square$ Trabajo no remunerado $\square$ Desplazamientos

Fuente: Ministerio de Empleo y Seguridad Social (2015).

\section{EL TRABAJO DECENTE EN EL MARCO DE LA AGENDA 2030}

La Agenda 2030 para el desarrollo sostenible, aprobada por Naciones Unidas en septiembre de 2015 (INE, 2019), abarca los tres ámbitos de la sostenibilidad: económica, social y medioambiental, y consta de 17 objetivos y 169 metas conexas, en cuyo objetivo número 8 se encuentra el trabajo decente, de cuya implementación se encarga la OIT a partir de los estudios y recomendaciones que dirige a los 187 Estados que la integran.

Los principales objetivos de la Agenda 2030 incluyen, junto al trabajo decente, los siguientes:

- La lucha contra la pobreza, que afecta 1.000 millones de personas, especialmente a mujeres y niños de Asia sudoriental y África subsahariana. 
- La lucha contra el hambre, que se manifiesta, por ejemplo, en que hay $800 \mathrm{mi}$ llones de personas subalimentadas y 70 millones de niños van al colegio con hambre.

- La promoción de la salud, para evitar que 5 millones de niños mueran antes de cumplir los 5 años, que en los países en desarrollo las mujeres solo reciban la mitad de la atención médica que necesitan, o que de los 40 millones de pacientes con sida que hay solo la cuarta parte tengan acceso a tratamiento con retrovirales.

- La promoción de la educación, para que disminuya la cifra de 60 millones de niños en edad de escolarización primaria que no asisten a la escuela o que 600 millones de jóvenes carezcan del nivel básico de aritmética y alfabetización.

- La igualdad de género, para impedir que 200 millones de niñas sigan sufriendo mutilación genital o para disminuir el 20\% de las mujeres que han sufrido violencia machista por parte de su pareja, sin que en la mitad de los países haya legislación que las proteja.

- El acceso al agua potable, ya que 1.000 millones de personas carecen de dicho recurso hídrico vital y 900 millones de personas defecan al aire libre.

- Las energías renovables y asequibles, para que hagan tomar conciencia de que más del $80 \%$ del consumo de energía es no renovable y aumenta diariamente el efecto invernadero.

- La innovación industrial generalizada, que evite que en los países en desarrollo solamente el 30\% de la producción agrícola esté mecanizada y el 16\% de la población mundial carezca de redes móviles de banda ancha.

- La reducción de las desigualdades, de forma que, por ejemplo, la inmensa mayoría de las personas con discapacidad en los países en desarrollo puedan hacer frente a los gastos sanitarios, al contrario de lo que sucede en estos momentos.

- Las ciudades sostenibles, ya que actualmente 3.500 millones de personas viven en ciudades y 900 millones lo hacen en barrios marginales, las ciudades ocupan el 3\% de la tierra y consumen el $80 \%$ de la energía, de forma que el $90 \%$ de los vecinos de las ciudades respiran aire insalubre y, a veces, peligroso.

- El consumo responsable en el campo de la alimentación y la energía, que advierta del grave problema que supone que haya 2.000 millones de personas con sobrepeso y que los hogares consuman el $30 \%$ de la energía y emitan el $20 \%$ del $\mathrm{CO}_{2}$, que contribuye al efecto invernadero.

- La lucha contra el cambio climático, ya que las emisiones de $\mathrm{CO}_{2}$ han aumentado un $50 \%$ desde 1990 . 
- La protección de la vida submarina, puesto que los océanos limpian el 30\% del $\mathrm{CO}_{2}$ producido y proporcionan proteínas a más de 3.000 millones de habitantes.

- La protección de los ecosistemas terrestres, ya que 2.600 millones de personas dependen de la agricultura y 1.600 millones, de los bosques, y la desertización avanza a 23 hectáreas por minuto.

- La Justicia como camino para la paz haciendo frente a la corrupción, que cuesta más de un billón de dólares, dicho sea en español, a los países en desarrollo, y el 30\% de los internados en instituciones penitenciarias está sin condena.

- El uso del diálogo, la cooperación y las alianzas para alcanzar estos objetivos.

Situándonos en el contexto mundial anteriormente descrito, podemos analizar la manifestación del trabajo decente en sus cuatro aspectos fundamentales: promoción del empleo, derechos de los trabajadores, protección social y diálogo social.

La primera característica del trabajo decente es que haya trabajo, que institucionalmente se promocione el empleo, y, en este sentido, la OIT aconseja a sus miembros la puesta en marcha de varios tipos de proyectos, como:

Una formación profesional altamente especializada y experimentada, adaptada a las necesidades de unas empresas que colaboren directamente en la formación de los estudiantes.

La formación permanente o educación a lo largo de toda la vida que ponga en valor y haga convertible cualquier tipo de conocimiento y experiencia y permita, gracias a su especialización y actualización, hacer unos trabajadores más polivalentes, que puedan encontrar trabajo, conservar el trabajo, reconvertirse en el trabajo, ascender en el trabajo y cambiar de trabajo.

La transformación de la política ocupacional clásica en talleres de empleo que identifiquen nichos de desarrollo económico, intensivos en mano de obra, y seleccione a unos estudiantes a los que formen, prácticamente integrándolos en proyectos de largo recorrido como trabajadores experimentados.

El fomento de la microfinanciación de las pequeñas empresas, que tejen la base socioeconómica de la sociedad y ocupan a una gran cantidad de mano de obra asalariada o se autoemplean como trabajadores independientes que tratan de adaptar su oferta a la demanda en condiciones muchas veces difíciles.

La segunda característica del trabajo decente tiene que ver con los derechos de los trabajadores, siendo conscientes de que el trabajo no es una mercancía y que su ejercicio es inseparable de la persona que lo presta; ya que, de lo contrario, el trabajador o trabajadora se encontraría en una situación de manifiesta debilidad respecto del emplea- 
dor que lo contrata si no fuera por su derecho a la sindicación o a la negociación colectiva, y al amparo del derecho del trabajo y los servicios de la Seguridad Social.

De entre los derechos de los trabajadores que garantizan un trabajo decente debemos subrayar:

El salario justo, adecuado a la prestación realizada y a su duración, que garantice la supervivencia del trabajador y su familia. Si el salario es insuficiente, provoca la existencia de trabajadores pobres (FOESA, 2019) que no llegan a fin de mes y, como están ocupados, no pueden formarse y buscar otro trabajo.

La jornada máxima de 8 horas diarias y 48 horas semanales, que tenga en cuenta los horarios de prestación, continuos o partidos, las horas extraordinarias remuneradas, los descansos diarios y semanales, las vacaciones anuales y la desconexión digital, de forma que una adecuada administración de la jornada de los trabajadores permita conciliar la vida laboral con la vida familiar.

Las condiciones de trabajo en un ambiente sano y seguro con un clima laboral humano e integrador.

Evitar el desempleo, el subempleo y el empleo informal redunda en los derechos de los trabajadores y permite contribuir fiscalmente con el Estado para que sea viable garantizar los servicios públicos y asistenciales.

En sociedades desarrolladas en las que abunda el trabajo precario, las alternativas que se plantean tienen que ver con la renta mínima de inserción, que pretende ayudar a las familias, reconvertir a los desempleados y crear una bolsa de personal disponible para tareas de emergencia social; y con la renta básica universal, que asegura a todos los ciudadanos unos ingresos suficientes para garantizar su consumo y su contribución fiscal, evitando situaciones sociales dramáticas y conflictos sociales que muchas veces pueden ser más costosos que lo invertido en dicha renta básica universal, aunque resulte difícil de financiar y sea previsible que se cree una cultura que desincentive el trabajo.

Como explica Susana de la Casa (2018: 656):

Las rentas mínimas de inserción son prestaciones que, aun teniendo en común varios rasgos con la renta básica universal, se diferencia de esta en tres cuestiones fundamentales: son medidas destinadas a luchar contra la pobreza, no se conceden a la generalidad de los individuos sino a la unidad familiar y se condicionan a la inserción social y laboral.

La tercera característica del trabajo decente tiene que ver con la protección social de los trabajadores, es decir, con el control de la seguridad e higiene en el trabajo desde los organismos del Estado; con los servicios sanitarios públicos, que en algunos países llegan al servicio de salud universal, como en España, y a determinadas prestaciones, entre las que podemos destacar las siguientes: 
Es un trabajo decente cuando hay control para evitar enfermedades profesionales y accidentes de trabajo, incluso in itinere, y cuando suceden, hay prestaciones para afrontarlos, incluidas bajas laborales retribuidas y periodos de rehabilitación.

Es un trabajo decente cuando los trabajadores tienen seguro de desempleo retribuido durante un periodo de tiempo proporcional al tiempo trabajado, prorrogable mediante prestaciones no contributivas en caso de necesidad.

La protección y ayudas a la contratación de trabajadores en riesgo de exclusión por ser jóvenes en busca de un primer empleo o por ser trabajadores mayores de 45 años con dificultades para reciclarse o para mantenerse en los puestos de trabajo.

Las prestaciones por jubilación son otro de los derechos típicos del trabajo decente, y se trata de una aportación económica que recibe el trabajador jubilado siempre y cuando haya cumplido un periodo de tiempo mínimo durante el cual haya contribuido y de cuya duración, categoría y cuantía de la contribución se deducirá la cuantía de la pensión que recibirá durante el resto de su vida, actualizándose en función de lo regulado en la normativa vigente. Esta actualización se ve afectada por la estimación de la esperanza de vida y el montante total de los perceptores de estas prestaciones.

Las prestaciones por maternidad y paternidad y la liberación del tiempo retribuido para atender al recién nacido son otra característica del trabajo decente, y, según los sistemas de seguridad social, las madres y los padres disponen de cierta cantidad de semanas antiguamente utilizadas solo por las madres y que, en el caso de España, recientemente deben utilizar también los padres para evitar la discriminación de género que a partir de esta circunstancia sufrían las mujeres.

La protección por viudedad u orfandad para los derechohabientes del trabajador o trabajadora fallecidos. En ambos casos la pensión se calcula como un porcentaje de la base reguladora y dura hasta los 25 años en el caso del huérfano que no trabaje y por el resto de la vida para el viudo o viuda.

La cuarta característica del trabajo decente tiene que ver con el diálogo social que toma cuerpo en varias manifestaciones como las siguientes:

- El derecho a la información, cotidiana, actualizada y veraz, de los trabajadores en su empresa y sobre su trabajo.

- La libertad sindical y el derecho de los trabajadores a afiliarse al sindicato que mayor confianza le merezca para la defensa de sus derechos laborales dentro y fuera de la empresa, ante las autoridades y ante las organizaciones patronales.

- El derecho de los trabajadores a elegir democráticamente a sus representantes, ya sea mediante órganos colegiados o unipersonales, en función del tamaño de la empresa. 
- El derecho de huelga, solamente limitado por determinadas circunstancias y garantizando unos servicios mínimos cuando se trate de servicios públicos como el transporte, la policía, la sanidad, las telecomunicaciones, etc.

- El derecho a la negociación colectiva autonormativa; es decir, el derecho a que lo acordado entre particulares, equilibrados por el carácter colectivo de la negociación entre trabajadores y empresarios, en plural, sea considerado una norma de obligado cumplimiento y en el marco del criterio de aplicar la norma mínima y la norma más favorable para el trabajador.

- La libertad sindical y el derecho a la negociación colectiva son los aspectos fundamentales del requisito del diálogo social como aspecto fundamental del trabajo decente.

A este planteamiento dialogante y negociador se suma el carácter tripartito de muchas negociaciones y acuerdos en los que interviene el Estado como otra parte que amalgama lo acordado entre empresarios y trabajadores, dándole un rango jurídico elevado y supervisando y vigilando su cumplimiento por las partes.

El trabajo digno, en definitiva, exige una constante política de empleo, la posible reivindicación judicial de los derechos de los trabajadores, la institucionalización de la protección social y el diálogo social acompañado de la libertad sindical y la negociación colectiva autonormativa.

\section{BIBLIOGRAFÍA}

Albizu, E. (1997). Flexibilidad laboral y gestión de los recursos humanos. Barcelona: Ariel.

Apgar, M. (2002). El lugar de trabajo alternativo: Cambiar el lugar y la manera de trabajar de la gente. Bilbao: Deusto.

Banco Mundial (01 de julio de 2019). World Bank. Obtenido de https://www.bancomundial.org/es/topic/poverty/overview

— (01 de julio de 2019). World Bank. Obtenido de https://datos.bancomundial.org/indicador/SL.TLF.TOTL.IN

Beck, U. (27 de noviembre de 2005). "La revuelta de los superfluos". El País.

Carnoy, M. (2001). El trabajo flexible. Madrid: Alianza.

Comisión de la Comunidades Europeas. (2006). Libro Verde para la modernización del Derecho laboral para afrontar los retos del siglo XXI. Bruselas: UE. Obtenido de https://eur-lex.europa.eu/legal-content/ES/TXT/?uri=celex:52006DC0708 
De la Casa, S. (2018). "Trabajo decente y garantía de una renta mínima de inserción”. En J. L. Monereo, El Trabajo decente (pág. 656). Granada: Comares.

Falguera, M. (2007). El Libro Verde para la modernización del Derecho del Trabajo de la Unión: una preocupante iniciativa. Cataluña: Lus Labor. Obtenido de https:// www.raco.cat/index.php/IUSLabor/article/view/72318

FOESSA (01 de julio de 2019). Cáritas. Obtenido de https://www.google.com/search?client=firefox-b\&ei=7CnZXNfeDaK5gwfF $25 \mathrm{eYAg} \& \mathrm{q}=$ foessa + trabajadores + pobres\&oq=foessa+trabajadores+pobres\&gs_l=psy-ab.3 ...2823.8782..9022...0.0..0.1 $05.2094 .24 \mathrm{j} 2$ 2..............gws-

Fundación $1^{\circ}$ de Mayo (01 de julio de 2019). Obtenido de http://www.1mayo.ccoo.es/ Publicaciones/Estudios_y_Proyectos/Informes\&21385

Fundación $1^{\circ}$ de mayo. (2019). Mercado de trabajo y protección por desempleo. Obtenido de http://www.1mayo.ccoo.es/Publicaciones/Estudios_y_Proyectos/Informes\&21385

Gil y Gil, J. L. (2008). "La flexiseguridad y el futuro del Derecho del Trabajo". Relaciones Laborales (223): 148-149.

Gobierno de España (01 de noviembre de 2019). Agenda 2030. Madrid. Obtenido de https://www.agenda2030.gob.es/

Igartúa, M. T. (2018). “Trabajo decente y conciliación de la vida laboral y familiar”. Ed Comares. Granada: en José Luis Monereo et. al.: El Trabajo Decente.

ILO (2015). ILO on global estimates on migrant workers. Obtenido de https://ilo.org/ wcmsp5/groups/public/---dgreports/---dcomm/documents/publication/ wcms_436343.pdf

- (2017). Global Estimates of Modern Slavery. Ginebra, Suiza. Obtenido de https:// www.ilo.org/wcmsp5/groups/public/@dgreports/@dcomm/documents/publication/ wcms_575479.pdf

- (2017). Global stimates of child labor. Obtenido de https://www.ilo.org/wcmsp5/ groups/public/@dgreports/@dcomm/documents/publication/wcms_575499.pdf

- (2019). El uso de robots industriales está aumentando rápidamente y tiene el potencial de modificar los procesos productivos en todo el mundo. Obtenido de https:// www.ilo.org/global/topics/future-of-work/trends/WCMS_618491/lang--es/index. htm

INE (2019). Instituto Nacional de Estadística de España. Obtenido de https://www.ine. es/jaxiT3/Datos.htm?t=4241

- (01 de julio de 2019). Instituto Nacional de Estadística de España. Obtenido de http://www.ine.es/jaxiT3/Datos.htm?t=4247

Miguélez, F. (2002). “¿Por qué empeora el empleo?”. Sistema, 168-169: 37-52. 
Ministerio de Empleo y Seguridad Social (2015). Encuesta nacional de condiciones de trabajo. Madrid: Gobierno de España. Obtenido de https://www.insst.es/documents/94886/96082/Encuesta+Nacional+de+Condiciones+de+Trabajo+6\%C2\%AA+EWCS/abd69b73-23ed-4c7f-bf8f-6b46f1998b45

Ministerio de Trabajo, Migraciones y Seguridad Social (2019). Ministerio de trabajo, migraciones y seguridad social. Obtenido de Anuario de Estadísticas Laborales: http://www.mitramiss.gob.es/es/estadisticas/index.htm

Monereo, J. L. (2018). El Trabajo Decente. Granada: Comares.

OCDE (1999). Perspectivas del empleo 1999, Ministerio de Trabajo y Asuntos Sociales. Madrid: Organización para la Cooperación y el Desarrollo Económicos.

- (1999). conferencia internacional del trabajo. Memorias al director general: Trabajo Decente. Oficina internacional del trabajo Ginebra.

OIT (2002). Informe sobre el empleo en el mundo 2001. Ginebra: Organización Internacional del Trabajo. Obtenido de La vida en el trabajo en la economía de la información

- (2019). Informe Mundial sobre Salarios 2018/2019. ILO. Obtenido de https://www. ilo.org/global/research/global-reports/global-wage-report/2018/lang--es/index.htm

- (2019). Perspectivas sociales y del empleo en el mundo. Ginebra: Organización Internacional del Trabajo. Obtenido de https:/www.ilo.org/wcmsp5/groups/public/--dgreports/---dcomm/---publ/documents/publication/wcms_713013.pdf

Portes, A. (2013). Sociología Económica. Madrid: CIS.

Prieto, C. (2002). "La degradación del empleo o la norma social del empleo flexibilizado". Sistema, 168-169. 